\title{
Coronal fractures of the body of the hamate: Two case reports
}

\author{
Paul A Binhammer MD FRCSC ${ }^{1}$, Jaggi Rao BSc ${ }^{2}$ \\ ${ }^{1}$ Division of Plastic Surgery, Sunnybrook Health Science Centre, University of Toronto, Toronto and \\ ${ }^{2}$ McMaster University, Hamilton, Ontario
}

\section{PA Binhammer, J Rao. Coronal fractures of the body of the hamate: Two case reports. Can J Plast Surg 1998;6(2):81-84.}

Coronal fracture of the body of the hamate with associated dorsal dislocation at the bases of the fourth and fifth metacarpals is rare. Two cases of this injury, treated with open reduction and internal fixation, are reported. As well, other reports of coronal hamate fractures are reviewed.

Key Words: Carpal bones, Carpometacarpal joint, Fractured hamate, Hamatometacarpal joint

\section{Fractures coronales du corps de l'os crochu}

RÉSUMÉ : La fracture coronale du corps de l'os crochu associée à une dislocation dorsale aux bases des quatrième et cinquième métacarpes est rare. Deux cas de telles blessures, traitées par réduction ouverte et fixation interne sont présentés ici. On propose en outre un survol d'autres rapports de fractures coronales de l'os crochu.

$\mathrm{F}$ ractures of the hamate were classified by Milch (1) into body fractures and hook fractures; fractures of the body of the hamate were subdivided into those occurring on the radial side and those occurring on the ulnar side of the hook. Bowen (2) presented a hamate fracture in the coronal plane and, therefore, added a third category - fractures to the body of the hamate. The two cases described in this report are examples of this rare type of fracture; both were associated with disarticulations at the base of the fourth and fifth metacarpals. Open reduction and internal fixation led to rapid recovery in terms of stabilization of the hamatometacarpal joint and restoration of hand function.

\section{Case 1}

\section{CASE PRESENTATIONS}

A 29-year-old, right-hand dominant woman was referred five days after punching a steel door with her closed right fist. She was seen by physicians at the emergency department of a lo-

Correspondence: Dr PA Binhammer, Sunnybrook Health Science Centre, H-210, 2075 Bayview Avenue, North York, Ontario M4N 3M5. Telephone 416-480-6731, fax 416-480-6800, e-mail p.binhammer@utoronto.ca cal hospital, where she was placed in a fibreglass splint after reduction of the fifth metacarpal.

Follow-up physical examination revealed minimal to moderate swelling and ecchymosis over the base of her right fifth metacarpal. X-rays revealed a hamate intra-articular fracture with the fifth carpometacarpal joint (Figure 1). Displacement was noted to be approximately 3 to $4 \mathrm{~mm}$. An open reduction was recommended to reduce the dislocated fragment of the hamate, and eight days after the injury, the patient underwent open reduction and internal fixation.

Maintenance reduction of the fifth metacarpal was achieved by two $1.1 \mathrm{~mm}$ Kirschner wires driven between the fourth and fifth metacarpals. The dislocated fragment of the hamate was positioned and fixed in place with a $0.7 \mathrm{~mm}$ Kirschner wire. Two $1.2 \mathrm{~mm}$ screws were then used with a plate over the fragment, and the $0.7 \mathrm{~mm}$ Kirschner wire was removed. The hand and wrist were immobilized by a volar plaster splint for three weeks, at which time it was replaced by a short-arm cast to immobilize the extremity for an additional three weeks. Six weeks after the operation, the Kirschner wires were removed (Figure 2). The patient returned to full activities by 12 weeks after surgery and was asymptomatic when seen at eight months postsurgery. 


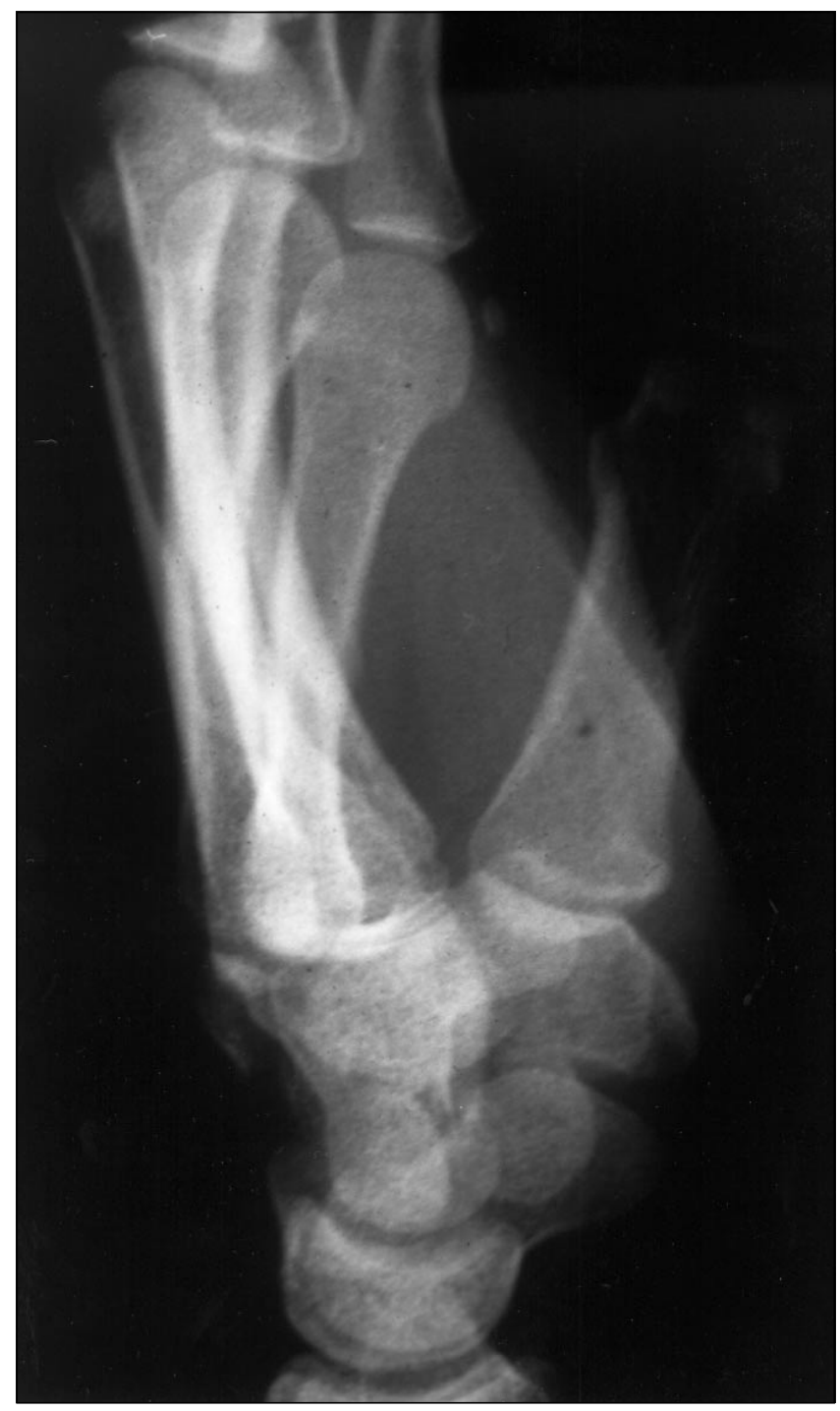

Figure 1) Case 1. Preoperative $x$-ray, lateral view

\section{Case 2}

A 34-year-old, right-hand dominant grocery clerk presented to the emergency department with pain on the dorsal ulnar aspect of his hand after a can of juice landed on the dorsum of his right hand. He was splinted and referred. Follow-up physical examination revealed swelling in the dorsum of his hand and maximal tenderness over the dorsum of the hamate. Radiological examination revealed intra-articular fracture dislocations of the ring and small carpometacarpal joints through the hamate and base of the ring metacarpal (Figure 3). Ten days after the injury, closed reduction percutaneous pinning was performed under fluoroscopic control. The hand and wrist were immobilized by a plaster splint.

A follow-up computed tomography scan of the hand indicated that the dorsal surface of the fifth metacarpal was unsupported by the hamate and that there was a gap at the articular surface at the base of the fourth metacarpal.

Twenty-two days after the injury, the patient underwent open reduction and internal fixation. At the time, a fracture was noted at the base of the fourth metacarpal in addition to

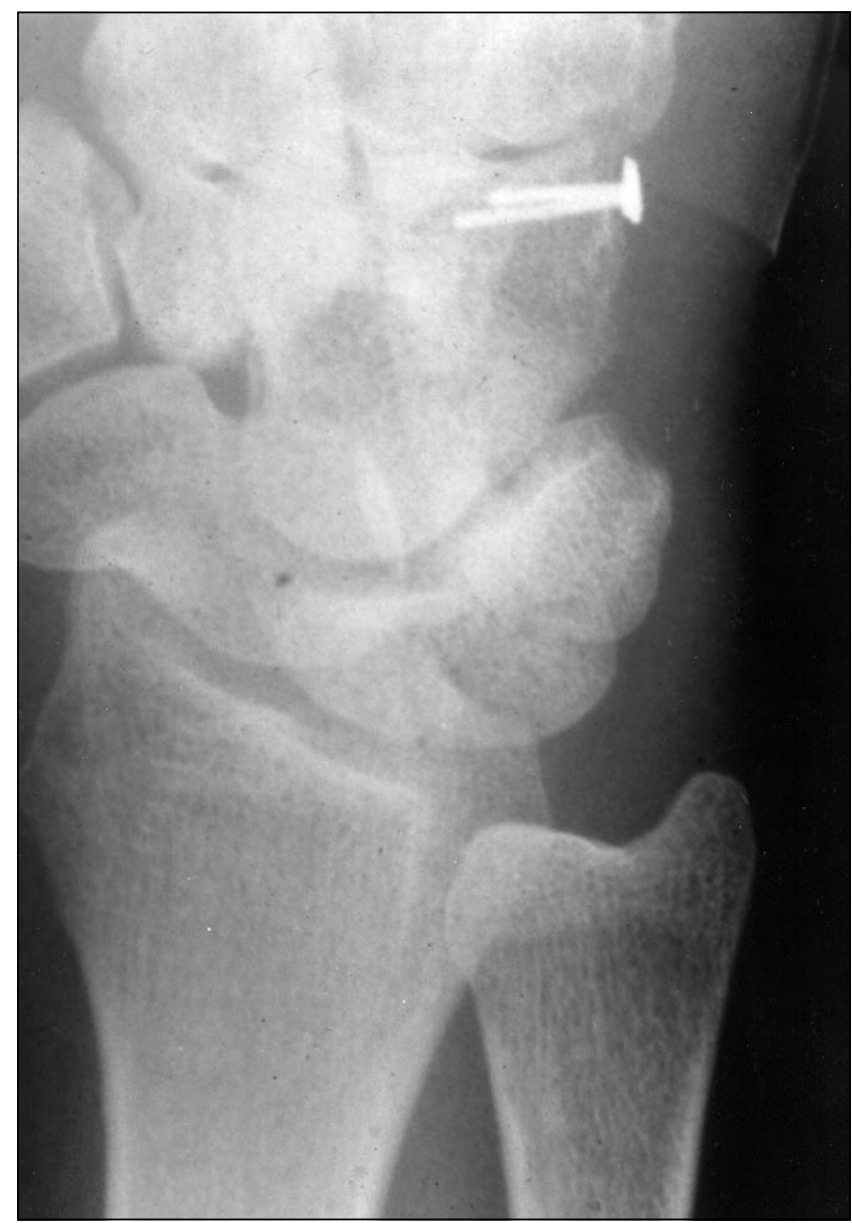

Figure 2) Case 1. Postoperative oblique x-ray showing the articular surface of the restored hamate

the hamate fracture (Figure 4). Reduction of the fourth and fifth metacarpals was achieved by passing a single Kirschner wire through the base of the fourth metacarpal dorsally into the capitate. The hamate fracture was then reduced into its appropriate position and maintained by a medium Leibinger hand plate with four screws (Figure 5). The patient's arm was immobilized and after two weeks was placed in a short-arm cast.

Eight weeks after his second surgery, the patient's cast was removed, and radiological films showed satisfactory alignment of all of his metacarpals by the Kirschner wire and proper fixation of the distal carpal row by the plate. Three months following the second surgery, he was asymptomatic and had returned to work. At his most recent follow-up of eight months, he continued to be asymptomatic.

\section{DISCUSSION}

Coronal fractures of the hamate have been previously classified by Ebraheim et al (3) into three types. The first of these is a type $\mathrm{A}$ fracture that passes through the centre of the body of the hamate in the coronal plane. A type B fracture involves a significant portion of the articular surface but in a more oblique fashion. Finally, a type $\mathrm{C}$ fracture is a fracture dislocation at the level of the small metacarpal, with the hamate associated within an avulsion fracture. 


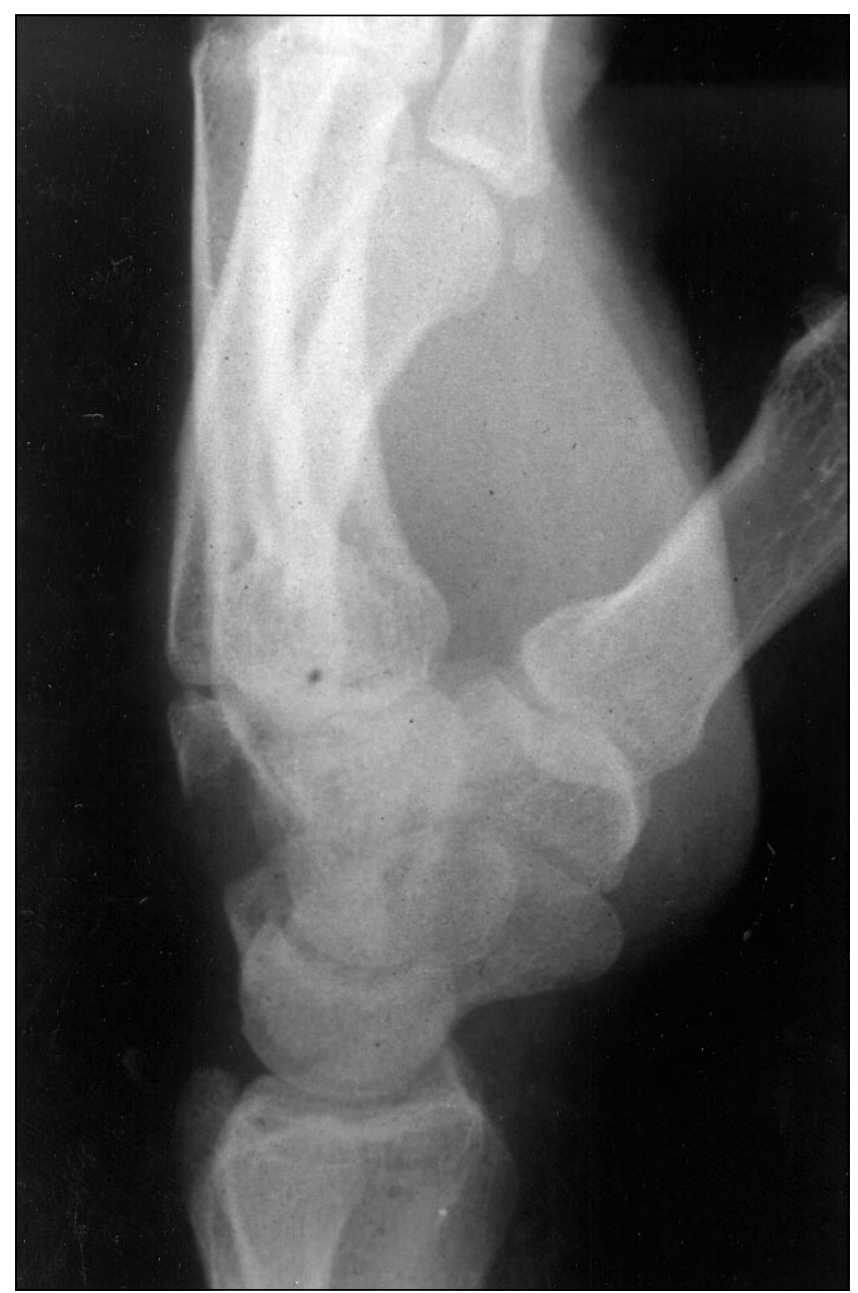

Figure 3) Case 2. Preoperative $x$-ray, lateral view

Without the availability of preoperative computed tomography scanning it may be very difficult to determine the difference between type A and B fractures. These types of fractures can be treated with open reduction internal fixation with Kirschner wires, Herbert screws and lag screws, or a combination of the above. It appears that the type of hardware required depends upon the fragment size.

The other important feature of operative treatment of the injuries is maintenance of the reduction of the dislocation that occurs at the fifth and possibly fourth carpometacarpal joint. This can usually be performed with the use of Kirschner wires.

Anatomical studies on cadaver wrists show that the obliquity of the fifth hamatometacarpal joint and the effect of the extensor carpi ulnaris and flexor carpi ulnaris tendons are responsible for the instability of coronal hamate fractures, causing proximal and ulnar displacement of the fifth metacarpal (3). The extensor carpi ulnaris inserts onto the dorsoulnar surface of the fifth metacarpal base, while the flexor carpi ulnaris exerts a proximal pull on the fifth metacarpal indirectly through the pisometacarpal ligament attached to the pisiform, onto which the tendon inserts. Displacement can

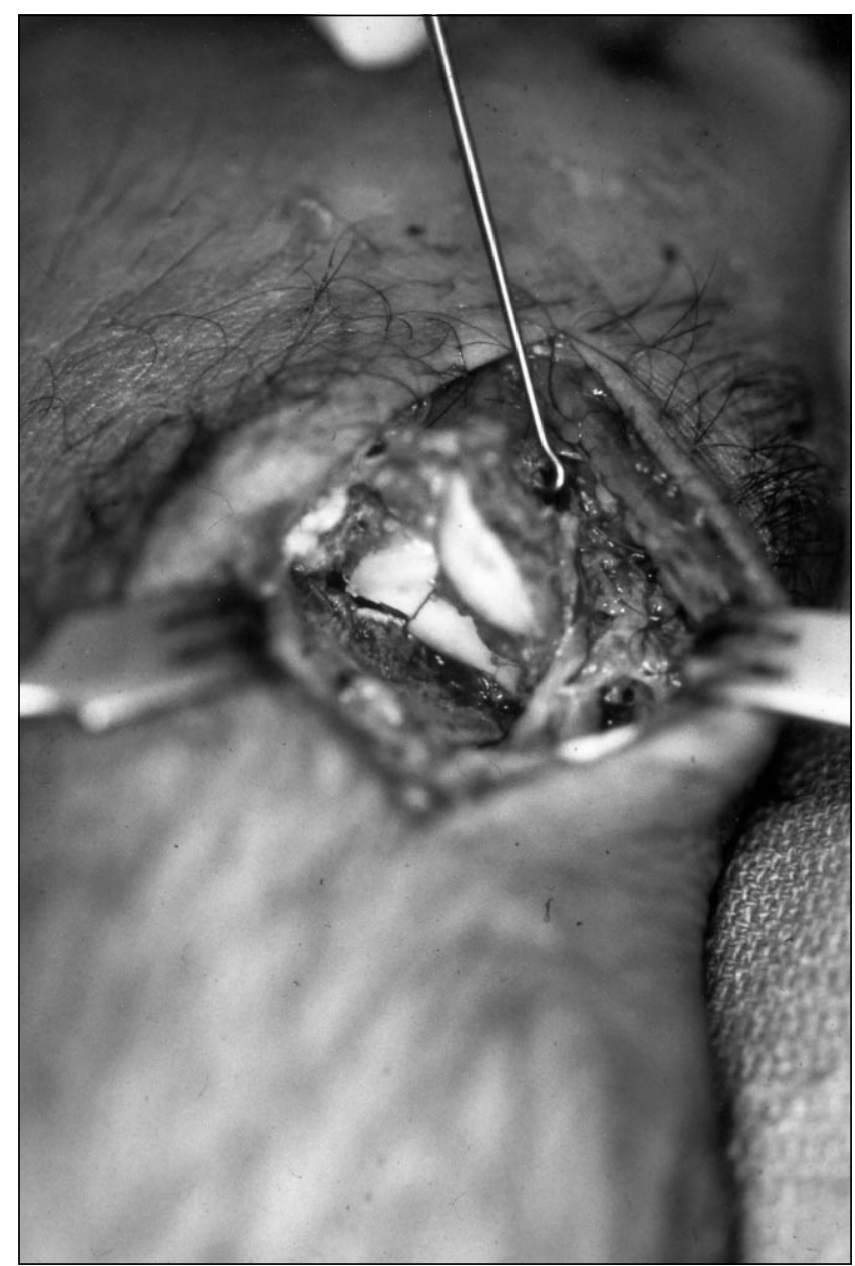

Figure 4) Case 2. Intraoperative photograph of the exposed dorsum wrist showing the minimally displaced fracture and the base of the fourth metacarpal with skin hook retracting the dorsal hamate fragment

also be caused by the pull of the hypothenar muscles, which originate partially at the hamatometacarpal joint. If internal fixation does not counter these forces, the hamatometacarpal joint is inadequately reduced, leading to muscle imbalance, loss of grip strength and painful arthritis.

It has been proposed that the mechanism of coronal hamate fractures involves transmission of an axial load through minimally flexed fourth and fifth metacarpals (4). On the basis of cadaver studies, Thomas and Birch (4) suggested that a force directed longitudinally along the metacarpals can split the hamate coronally if the wrist is in ulnar deviation because this position causes the proximal side of the hamate to abut against the triquetrum. They also showed that wrist palmar flexion could produce dorsal displacement of the hamate fracture fragment. In case 1, as well as in another case reported in the literature (5), where the patient punched a resistant object with a closed fist, the usual result is a boxer's fracture. However, it has been demonstrated that coronal fractures of the body of the hamate can occasionally occur when the wrist is ulnar deviated and palmar flexed (5) at the start of a punch. In case 2, the force that caused the fracture was an object that fell on the hand. It is likely that the impact 


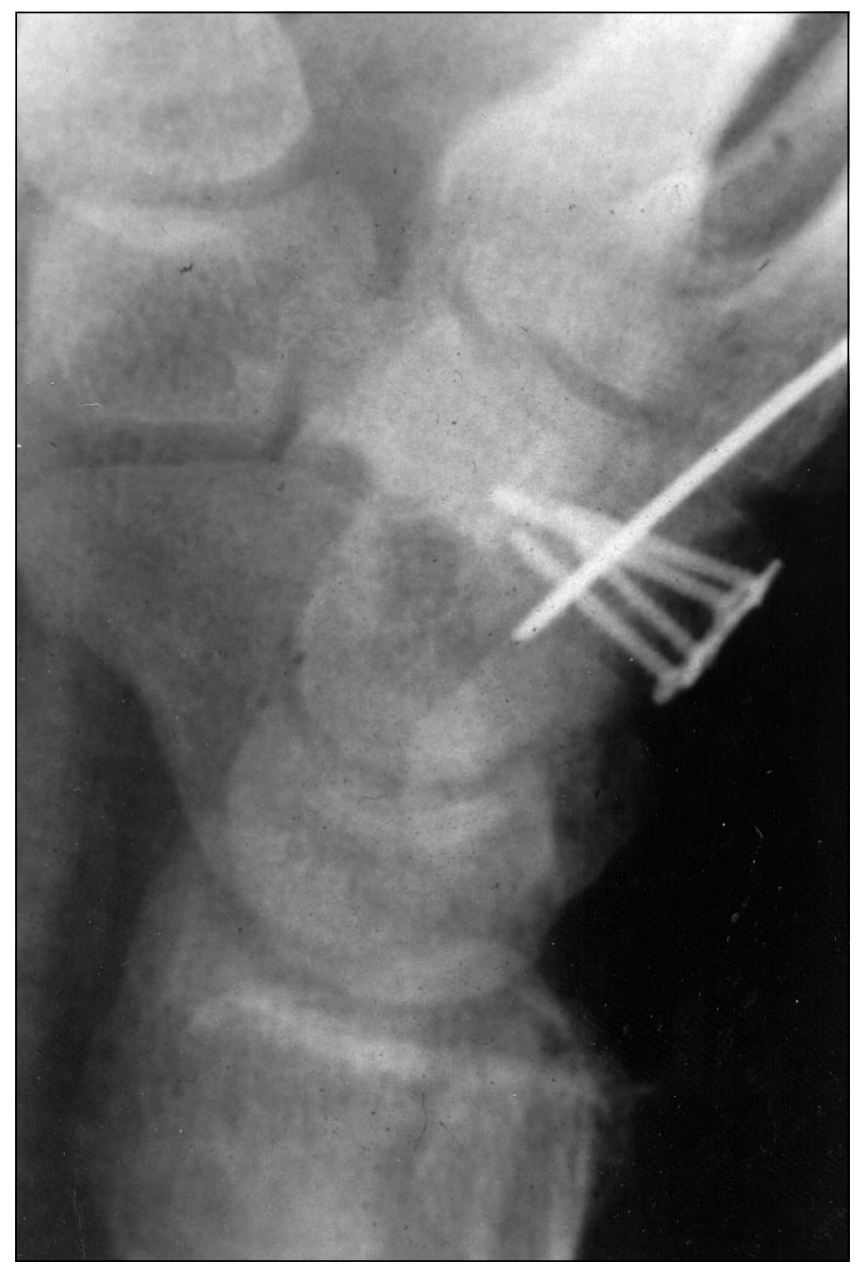

Figure 5) Case 2. Postoperative $x$-ray, lateral view, showing the placement of the Kirschner wire and four screws with a plate securing the hamate

was transmitted longitudinally along the metacarpals to fracture the hamate coronally. Loth and McMillan (6) also described a coronal hamate fracture caused by the impact of an object on the dorsum of the hand.

Coronal fractures of the hamate may be missed (4-8). This is because not all fractures are apparent from routine $\mathrm{x}$-ray views of the wrist. As well, infrequency of the injury and the absence of obvious clinical findings such as crepitus, de- formity and massive swelling contribute to the difficulty in detection. As such, diagnosis has been delayed as long as three months postinjury in some cases (4). It has been suggested that when a boxer's fracture is not found after a significant punching injury, fracture of the hamate should be suspected (3). Pain at the ulnar side of the hand in the absence of routine radiological findings, as well as fractures of the fourth metacarpal base, should warrant an oblique x-ray. Radiographic views of the forearm pronated at $30^{\circ}$ have been shown to profile the hamatometacarpal joint clearly and to define coronal hamate fracture and associated joint subluxation most effectively (9). Tomograms are helpful, particularly if the fracture is complex, involving multiple carpal bones in the fracture process (3).

In summary, two cases of an uncommon dorsal hamate carpometacarpal fracture-dislocation were treated by open reduction and internal fixation. Reduction of the dorsal displacement of the fourth and fifth metacarpal bases was easily maintained after reduction and fixation with Kirschner wires; the hamate fragment was reduced and fixed with a Kirschner wire in one case and with a plate with cortical screws in the other. The patients have demonstrated excellent results after an eight-month average follow-up.

\section{REFERENCES}

1. Milch H. Fracture of the hamate bone. J Bone Joint Surg 1934;16:459-62.

2. Bowen TL. Injuries of the hamate bone. Hand 1973;5:235-8.

3. Ebraheim NA, Skie MC, Savolaine ER, Jackson WT. Coronal fracture of the body of the hamate. J Trauma 1995;38:169-74.

4. Thomas AP, Birch R. An unusual hamate fracture. Hand 1983;15:281-6.

5. Kimura H, Kamura S, Akai M, Ohno T. An unusual coronal fracture of the body of the hamate bone. J Hand Surg 1988;13A:743-5.

6. Loth TS, McMillan MD. Coronal dorsal hamate fractures. J Hand Surg 1988;13A:616-8.

7. Roth JH, de Lorenzi C. Displaced intra-articular coronal fracture of the body of the hamate treated with a Herbert screw. J Hand Surg 1988;13A:619-21.

8. Marck KW, Klasen HJ. Fracture dislocation of the hamatometacarpal joint: A case report. J Hand Surg 1986;11A:128-30.

9. Freeland AE, Finley JS. Displaced dorsal oblique fractures of the hamate treated with a cortical mini lag screw. J Hand Surg 1986;11A:656-8.

10. Takami H, Takahashi S, Hiraki S. Coronal fracture of the body of the hamate: case reports. J Trauma 1992;32:110-2. 